\title{
The Required Cultivation on Chinese Character Structure of Teacher Teaching Chinese to Foreign Students
}

\author{
Lingzhi Zhang \\ Overseas Education College \\ Xiamen University \\ Xiamen, China \\ Yanmei Zhang \\ School of Foreign Language \\ Guilin University of Electronic Technology \\ Guilin, China \\ College of Liberal Arts \\ South China Normal University \\ Guangzhou, China
}

\author{
Han Ding \\ School of Foreign Language \\ Guilin University of Electronic Technology \\ Guilin, China \\ $\mathrm{Hu} \mathrm{Ye*}$ \\ School Of Journalism and Communication \\ Xiamen University \\ Xiamen, China \\ *Corresponding author
}

\begin{abstract}
This article introduces the cultivation on Chinese character structure that teachers teaching Chinese to foreign students should possess such as pictographs, indicative characters, associative compounds and phonograms. It indicates that only when teachers teaching Chinese to foreign students deeply understand the principle of Chinese character formation, can they pass on the knowledge of Chinese character to learners in more excellent manner. Besides, the practice widely opens the magical world behind characters to foreign students, letting them know how and know why so as to accomplish efforts yielding twice the result with half the effort
\end{abstract}

Keywords-teacher teaching Chinese to foreign students; Chinese character structure; innovative personnel; pictograph; indicative character; associative compound; phonogram

\section{INTRODUCTION}

Global Chinese language teacher, that is, mainland China constantly equals to 'teacher teaching Chinese to foreign students'. Concerning what kind of knowledge structure and competence that teacher teaching Chinese to foreign students should obtain, NOCFL (Confucius Institute Headquarters) officially released Standards for Teachers of Chinese to Speakers of Other Languages in 2007. In 2012, it issued formally Standards for Teachers of Chinese to Speakers of Other Languages (2012 version) (hereinafter referred to as 'new Standards'). New Standards revised based on the standard of the year 2007, lasting three years that thousands of experts at home and abroad and front-line teachers had taken part in its revision. The spotlight of new Standards is that it makes a specific request to the knowledge, ability and quality that teacher teaching Chinese to foreign students ought to accomplish.
Inside new Standards, with regard to Chinese teaching, Standards for Teachers of Chinese to Speakers of Other Languages (2007 version) has specific and detailed requirements to the knowledge structure and competence that teacher teaching Chinese to foreign students should own, and that is 'in Chinese teaching, teachers are supposed to comprehend the basic knowledge of Chinese characters, with the abilities and skills of Chinese characters knowledge to the learners.' Its specific performances are divided into two parts. Initially, teachers should grasp the following five basic principles.

- Teaching according to principles of Chinese characters formation.

- Pay attention to form, sound and meaning combined.

- Value its practicability and interest.

- Emphasis on teaching principles such as writing after the first recognized, commonly used words first, and compulsive repetition.

- Select useful and efficient Chinese characters teaching method in connection with learners.

In the next place, teachers ought to master the following eight foundational capabilities:

- Familiar with and grasp the basic features of Chinese characters and the knowledge, knowing and using relevant concepts and terms properly.

- Understand the composition of Chinese commonly used words and other words. 
- Formulate different Chinese teaching programs on learners of diverse learning objectives and levels.

- Comprehend learning needs and difficulties of the main Chinese language learners, can be targeted to carry out Chinese characters teaching.

- Understand the importance of Chinese teaching to Chinese learning, know the basic steps, rules and goals of Chinese teaching, and utilize the knowledge to effectively guide the Chinese teaching practice.

- Realize the inter-relationship of Chinese characters and Pinyin in Chinese language teaching, familiar with Chinese term relationships, reading and writing Chinese characters sequence.

- Get familiar with and obtain common teaching strategies, methods, techniques of Chinese teaching.

- Have the Chinese teaching ability of comprehension, development and innovation on different learners and learning environment.

New Standards in 2012 still clearly put forward that global Chinese language teachers have responsibilities to 'possess the analytical ability of basic Chinese phonetics, words, grammar and characters'. Besides, it also demands that teachers should 'master the fundamental principles and main contents of Chinese phonetics, words, grammar and characters teaching, grasp their methods and skills as well as use appropriate teaching methods according to different teaching objects'.

We consider that within the principles and abilities teachers teaching Chinese to foreign students should master when teaching Chinese characters, first and foremost is that teachers teaching Chinese to foreign students must memorize the make-up of Chinese characters (namely principle of Chinese characters formation) by heart and use them familiarly in teaching.

Chinese character is one of the oldest characters in the world. With the history of over 5000 years, Chinese nation has always used Chinese characters to record Chinese language as well as express thoughts. Chinese nation not only notes history and culture of Chinese nation of 5000 years, but as it is part of Chinese culture, it reflects specific mindset and aesthetic taste when recognizing things with its particular structure and pattern. In the case of teaching Chinese characters and spread Chinese character culture to overseas students, teachers themselves need to understand Chinese characters together with structure and formation principle.

\section{THE REQUiRED CULTIVATION ON CHINESE CHARACTER STRUCTURE OF TEACHER TEACHING CHINESE TO FOREIGN STUDENTS}

In that case, what kind of required cultivation on Chinese character structure should teacher teaching Chinese to foreign students possess? It is fair to say that the following four aspects are necessary:

\section{A. Teacher Teaching Chinese to Foreign Students Should Familiar with the Principle of Pictograph Formation in Chinese Character}

In the first instance, teacher teaching Chinese to foreign students should familiar with the principle of pictograph formation in Chinese character.

Pictograph can be classified into two types, single pictograph and compound pictograph.

1) Single pictograph: Single pictograph, namely uses the whole character to depict the shape of an object, easily showing its representative body, such as '日' ('sun'), '月， ('moon'), ‘山' ('mountain'), ‘水' ('water'), ‘木' ('wood'), ‘人’ ('human'), ‘女’ ('woman’), '目' ('eye’), ‘耳’ ('ear'), ‘口’ ('mouth') and so on.

2) Compound pictograph: The concept of compound pictograph is that it uses a symbol to depict the shape of an object while regarding another symbol as a foil to represent the object and synthesizes two symbols to one character. For instance, ‘眉' ('eyebrow'), ‘胃'('stomach'), ‘瓜'('melon') and '果'('fruit').

For example, the following characters '女' ('woman'), '人' ('human'), ‘燕' ('swallow') and '目' ('eye') use the principle of pictograph formation. From the pictures we can see the object it stands for soon. Character '女'('woman') is similar to a woman sits down on her heels with her hands crossed. "Fig. 1" Character '人'('human'), looks as if a person leans to one side. "Fig. 2" Character ' 燕, ('swallow') seems like a bird called 'swallow' and it highlights the unfolded wings. "Fig. 3" Character '目' ('eye') resembles eyes of a person. "Fig. 4"

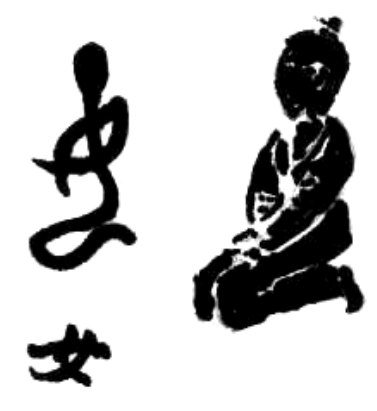

Fig. 1. pictograph: '女'

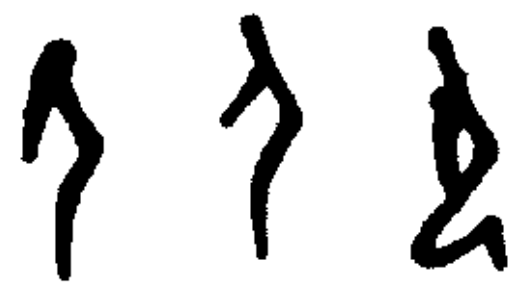

Fig. 2. pictograph: '人’ 


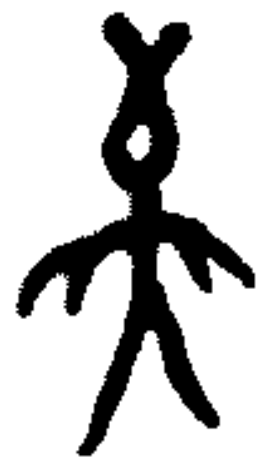

Fig. 3. pictograph: ‘燕’

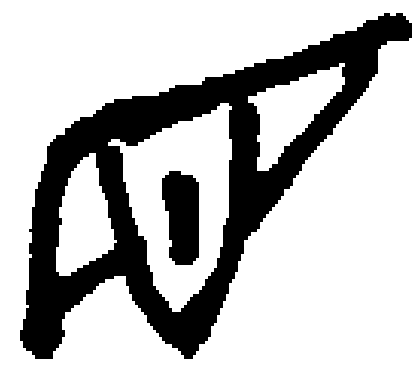

Fig. 4. pictograph: '目”

B. Teacher Teaching Chinese to Foreign Students Should Familiar with the Principle of Indicative Character Formation in Chinese Character

Secondly, teacher teaching Chinese to foreign students should familiar with the principle of indicative character formation in Chinese character.

Indicative character can be divided into two types. One type directly made of abstract symbols, like '上’ , ‘中' ,

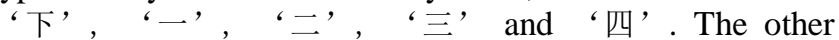
changes based on pictograph, from which it adds abstract symbols, deletes symbols, overturns characters or expresses abstract concepts and complex meaning, such as '本', ‘末”, ‘母’, ‘曰’, ‘甘’, ‘后’ , ‘司’ and so on.

For instance, the following case is the principle of indicative character '母' formation, modifying based on the pictograph '女'. In the picture below, a woman sits down on her heels with her hands crossed. "Fig. 5" It is the pictograph '女'. Subsequently, adding two dots to '女', it represents a woman nursing a baby, and that is the image of a mother. It is the indicative character '母'.
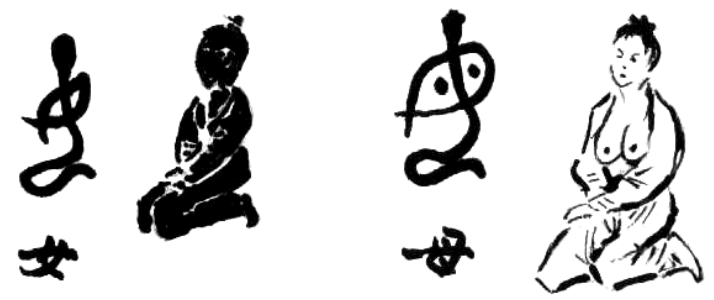

Fig. 5. The indicative character '母' changes based on the pictograph '女”

Pictograph and indicative character are both single characters. On the basis of pictographs, characters can be not only changed into indicative characters but made up of associative compound and phonogram.

\section{Teacher Teaching Chinese to Foreign Students Should Familiar with the Principle of Associative Compound Formation in Chinese Character}

Thirdly, teacher teaching Chinese to foreign students should familiar with the principle of associative compound formation in Chinese character.

The so-called associative compound usually combines two or several pictographs. That is to say, put two or several pictographs together to join their meaning. Associative compound is parceled into overlapped associative compound and variant associative compound.

1) Overlapped associative compound: Overlapped associative compound consists of two or several same forms, like ‘从’, ‘北’, ‘众’, ‘林”, ‘森” and ‘卉”. Although it is overlapped from the same symbol, it must represent a fresh sense; otherwise it cannot belong to associative compound.

2) Variant associative compound: Variant associative compound is made up of two or several different forms, such as '及”, ‘保”, ‘即’, ‘既”, ‘休” and so on.

Many associative compounds own strong pictographic so that their ancient writings resemble pictures. For instance, the following graphs are respectively the principles of associative compound formation '林' (overlapped associative compound) and ‘即' (variant associative compound).

The first character '林', namely two trees stand side by side, indicating the meaning of woods. "Fig. 6" The other one “即' seems a person move towards a utensil containing food. Thus, its original meaning is a person eating food, while its extended meaning is 'approach'. "Fig. 7" 


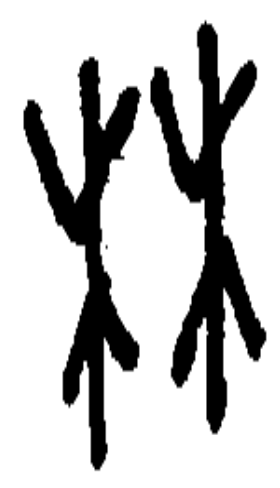

Fig. 6. Overlapped associative compound: ‘林’

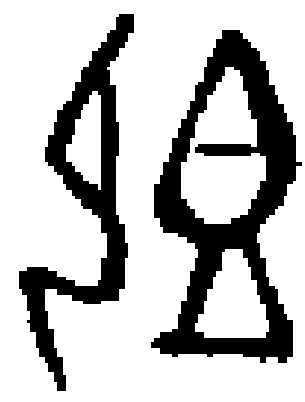

Fig. 7. Variant associative compound: ‘即'

\section{Teacher Teaching Chinese to Foreign Students Should Familiar with the Principle of Phonogram Formation in Chinese Character}

Fourthly, teacher teaching Chinese to foreign students should familiar with the principle of phonogram formation in Chinese character.

Phonogram, in common with associative compound, is also often the combination of two or several pictographs. However, unlike associative compound, part of it is not involved in signification. If we consider that the phonogram is made up of two parts, one part representing the category of sense and word meaning is called 'shape symbol' (or sentimental symbol, meaning symbol and semantic radical). The other can be roughly phonetic, namely 'phonological symbol' (or phonetic radical). It can be not only taken too literally, but taken too superficially to hear.

Phonogram breaks through the limitation of single signifying meaning of pictograph, indicative character and associative compound. In addition, it combines the pronunciation and meaning together as well as improves the adaptability of characters to the language, which is the most productive character mode. Shuo Wen Jie Zi contains 9353 characters in total, including 7700 phonograms, accounting for 82 percent.

According to different positions of phonogram shape symbol and phonological symbol, we can sum up its formation mode into eight types. They are respectively phonetic part on right side, phonetic part on left side, phonetic part on lower side; phonetic part on upper side, phonetic part inside, phonetic part outside, phonetic part in the corner and semantic part in the corner. Nevertheless, it is the positions of shape symbol and phonological symbol that have abnormal conditions, like phonetic part on left side, phonetic part on right side, phonetic part outside, phonetic part inside, phonetic part on lower side, phonetic part on upper side. Shape symbol only represents the large class of this character and just in terms of original meaning. Phonological symbol can sometimes be phonographic and express meaning, and these are characters of associative compound with phonogram, such as ‘驱’, ‘娶’, ‘婚” and so on.

The ideographic function of phonogram semantic radical can prompt a great deal of information on Chinese culture. Taking radical '女' as an example. Since ancient China once had an age of matriarchal society, the majority of surnames that time added radical '女” like ‘姜', ‘姚”, “姬” and ‘嬴”. The character '姓' itself has radical '女”. Concerning marriage, many characters have radical '女', such as '嫁”, '娶', ‘姻', ‘媒', ‘妁' and so on. When it comes to the time of patriarchal society, it can be seen from the characters that

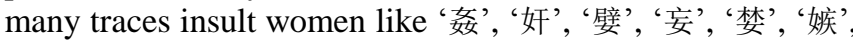
'嫌' and '妨'. Among them, some characters now have been modified to another style of writing. For example, '媮' has changed to '偷' and '嬾' has converted into '懒'

Furthermore, it might also be noted that there are some limitations in phonetic function of phonogram phonetic radicals. It is recorded in Xinhua Dictionary that 39 percent of phonetic complements are efficient (with identical consonants, vowels and tones). At present, owing to inaccurate phonetic function of phonogram phonetic radicals, we probably afraid to read the character according to its phonetic radical when coming up to a rusty phonogram. For instance, there are plenty of phonograms using '工' as their phonetic radicals, like '缸', ‘杜', ‘肛' and '江', with diverse initials ' $g$ ', ' $k$ ', ' $h$ ' , 'j', 'q', ' $x$ '. In fact, during the period of producing characters, the pronunciations of characters mentioned above are quite close. However, it is the changes of sounds in subsequent dynasties that cause divergences of their pronunciations. And that is why the saying 'a scholar recognize a character by reading half the character' unreliable. According to statistics, since the evolution of ancient and present sounds, approximately three-quarter phonograms possess not identical pronunciations of their phonetic radicals and whole characters. This is also the confinement of phonetic function of phonogram phonetic radicals.

As a teacher teaching Chinese to foreign students, it is particularly important to note that as mentioned above, 'Shuo Wen Jie Zi contains 9353 characters in total, including 7700 phonograms, accounting for 82 percent.', namely about 80 percent of Chinese characters are phonograms. Therefore, Chinese character teaching of TCSL emphasizes on phonograms. It is available to make clear meaning of radicals when helping students learning Chinese to comprehend many characters. Simultaneously, though the saying 'a scholar recognize a character by reading half the character' may not be reliable, quite a few phonological symbols of phonograms still lend hands with students a fine prompt action in terms of character and pronunciation. 


\section{CONCLUSION}

In brief, we hold the opinion that the formation principles of pictographs, indicative characters, associative compounds and phonograms are the necessarily required cultivation on Chinese character structure of teacher teaching Chinese to foreign students. The cultivation demands teachers to master the basic mode of Chinese character structure as well as respective characteristics of pictographs, indicative characters, associative compounds and phonograms. It is especially necessary for teacher teaching Chinese to foreign students to learn the make-up of Chinese characters; strengthen acquaintance to the relation of sound, form and meaning of Chinese character and improve the ability to read, utilize and teach Chinese character.

Only when teacher teaching Chinese to foreign students tap into the principle of Chinese character formation, can they better impart knowledge of Chinese characters to learners. Apart from this, Chinese language teachers open a wonderland behind characters to the students, allowing them to feel the picture fascination of hieroglyphs and experience the profound word principles of indicative characters, associative compounds and phonograms. Moreover, teachers have the ability to help students to link with sound, form and meaning of characters in order to achieve the effect of yielding twice the result with half the effort that students understand how and why.

\section{ACKNOWLEDGEMENT}

This research was financially supported by Fujian Social Science Planning Fund Program \& Annual key projects of China Special Socialism Theory System Research Center in Fujian ( 2012B237).

\section{REFERENCES}

[1] Li Wang (Chief Editor). Classic Chinese (Revised Edition) (four books) [M]. Beijing: Chinese Publishing House, 1999.

[2] Bingjun Zhou. Ancient Chinese Outline [M]. Changsha: Hunan Education Press, 1981.

[3] Qinge Liu. Ancient Chinese Crash Reader [M]. Beijing: Chinese Publishing House, 2002.

[4] (Han Dynasty) Shen Xu (Editor), (Song Dynasty) Xuan Xu (Proofreader). Shuo Wen Jie Zi [M]. Beijing: Chinese Publishing House, 2013. 